\title{
Brain-Stem and Cortical Interactions in the Interhemispheric Delayed Response
}

\author{
L. T. Rutledge and Thelma T. Kennedy ${ }^{1}$ \\ Department of Physiology, University of Michigan Medical School, Ann Arbor, \\ Michigan, and Department of Physiology and Biophysics, University of Washington \\ School of Medicine, Seattle, Washington
}

Received August 17, 1961

\begin{abstract}
Acute experiments on cats under chloralose anesthesia were designed to study the role of the brain stem in the transmission of the interhemispheric delayed response (IDR). Experiments on interaction of potentials recorded in brain stem and at cortex led to the conclusion that the IDR is relayed to the contralateral hemisphere via routes which pass through the general area of the mesencephalic reticular formation. Other brain-stem areas, not directly in the pathway, also have access to the cortical fields discharging the IDR. During passage through the mesencephalon and elaboration at the cortex the IDR comes under the influence of afferent activity. To a more or less considerable extent, depending on the time relationships, this interaction is reciprocal. Characteristics of the IDR were investigated and the findings confirmed in preparations with brains radically split longitudinally in the mid-line. An integrative function based upon cortical-brain-stem-cortical and afferent activity is suggested for the IDR system.
\end{abstract}

\section{Introduction}

A late wave following the transcallosal response (TCR) was identified and named the "interhemispheric delayed response" (IDR) in a previous publication (11). The IDR was described as representing a system, multisynaptic in nature, showing considerable lability, susceptible to "habituation" effects, and most easily studied under moderate systemic chloralose. Neither callosal nor commissural pathways mediated the response; thus it appears that deeper, subcortical structures are implicated in the interhemispheric transmission. A subsequent report (12) outlined brain-stem areas involved in the IDR relay and suggested how this

1 Aided by grants 2-B-5082 and B-2978 from the National Institute of Neurological Diseases and Blindness, and Senior Research Fellowship SF-122 from the U. S. Public Health Service. 
information-carrying system might function in an integration of activity between cortex and brain stem.

Evidence to be presented substantiates our suggestion that the IDR is relayed through brain-stem areas, and that activity in this pathway appears to influence nonspecific afferent systems and can, in turn, be modified by afferent activity. It will also be shown how this pathway is operative in a brain radically separated in the mid-line from the anterior pole to the mesencephalon.

\section{Method}

The experimental technique was that described in the first publication (11), wherein potentials evoked by cortical stimulation were recorded from the exposed contralateral hemisphere. Brain-stem stimulation and recording via stereotaxically-placed electrodes, and a study of five cats whose brains had been radically "split" in the mid-line several months previously, were new additions for the present work. Twenty-seven cats were studied, all under an initial dose of 40 to $55 \mathrm{mg}$ per kilogram chloralose, and frequently prepared under a short-acting barbiturate or ether. To elicit consistent, maximum amplitude responses for interaction purposes, suprathreshold intensities of single shock stimulation, 0.1- to 0.5 msec duration, were usually employed. For the cortex, these intensities were frequently approximately one-fourth higher than that necessary for a maximum TCR. Voltage actually varied between 10 and 38 among the various animals, and although current was not monitored, it was estimated to have been generally less than $1.5 \mathrm{ma}$ for cortical, and probably much less than 1.0 ma for brain-stem stimulation. Depth electrodes were concentric bipolar, with an insulated nichrome wire for monopolar recording enclosed in 22-gauge insulated stainless steel tubing. Stimulation was between the two exposed tips, about $0.5 \mathrm{~mm}$ apart. At the conclusion of an experiment, current was passed through the stainless steel electrode for histological identification of its locus, by means of the Prussian blue method.

\section{Results}

IDR Relay and Interactions. A brain-stem structure or area may be assumed to be a relay in the IDR pathway if: $(a)$ an evoked response can be recorded there following cortical stimulation, $(b)$ stimulation there produces an evoked cortical response, and $(c)$ occlusion of the IDR can be demonstrated following stimulation of the brain-stem area.

Because of the facilitated neuronal transmission in the chloralosed 
preparation $(4,7)$ criteria $(a)$ and $(b)$ alone usually cannot be used to define a "relay." With low to moderate intensities of stimulation to any of several brain-stem areas, it is extremely easy to produce a variable evoked response at the cortical surface, which both resembles the IDR and can be occlusively interacted with it. Thus, the important criterion often becomes the evidence of evoked response in the depth structure to cortical stimulation.

Figures have been constructed to illustrate only the salient features of brain-stem recording, stimulation, interactions, and other parameters. The variable wave forms of the evoked responses in depth, the large number of brain-stem areas studied, and the complex interactions as seen on simultaneous multiple oscilloscope recordings, make it virtually impossible to discuss all data.

In the upper left frame of Fig. 1 are responses to cortical stimulation

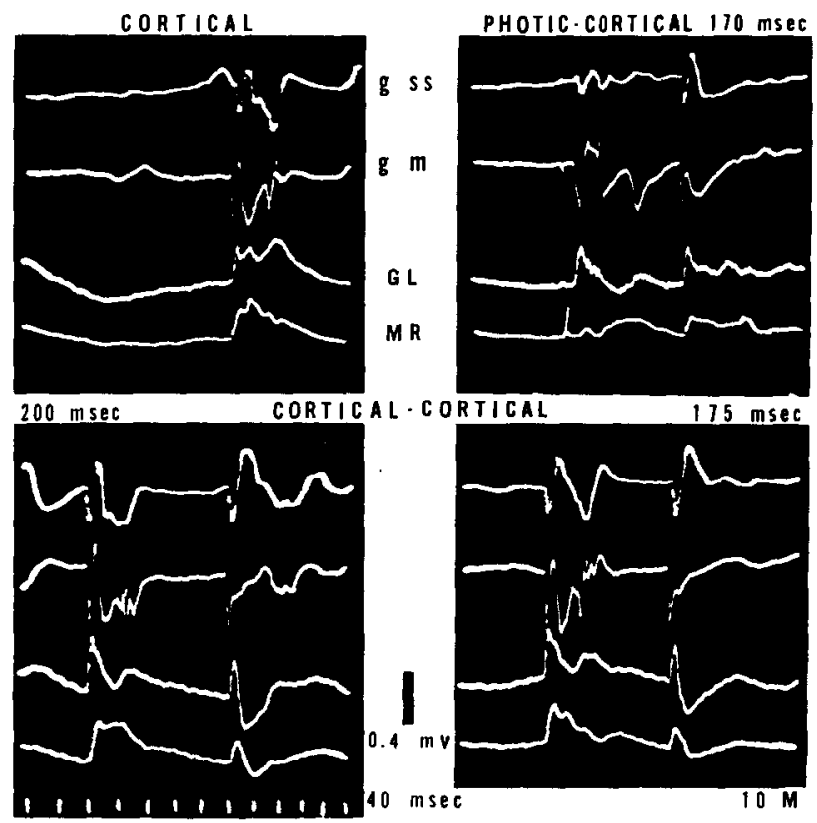

FIG. 1. Interactions, four-beam records. Cortical stimulation of left gyrus midsuprasylvius at $A 9$, recordings from right gyrus suprasylvius at $A 7$ ( $g$ ss), right gyrus marginalis at $\mathrm{Al}(\mathrm{g} \mathrm{m})$, left lateral geniculate (GL) at A6.5, L9.0, +3, and right mesencephalic reticular formation (MR) at A3.5, R2, -0.5 (GL and MR sites histologically verified). Photic stimulation to both dilated pupils with 5-msec flash, GE No. 27 bulb driven by Grass Stimulator. Stimulus artifacts appear as breaks in baseline, but sweeps not perfectly aligned. 
as simultaneously recorded on the contralateral gyrus suprasylvius ( $\mathrm{g}$ ss), on the contralateral gyrus marginalis $(\mathrm{g} \mathrm{m})$, in the ipsilateral lateral geniculate body (GL), and in the contralateral mesencephalic reticular formation (MR). The initial waves in the cortical records are the TCR followed by the largely positive IDR at approximately $60-\mathrm{msec}$ latency to the peak. Responses in GL and MR are irregular, attenuated negative waves with the earliest negative peaks at about $30 \mathrm{msec}$. Though these responses in depth are typical, in some experiments, and especially in MR locations, the recorded event was primarily positive. To ascertain refractory times of these responses, dual cortical shocks were utilized, as represented in the two lower frames at conditioning-test times of 200 and $175 \mathrm{msec}$. Block of IDR of approximately 50 per cent and 100 per cent, respectively, are represented by these times. In both depth locations there is the appearance of a positive wave with the disappearance of the attenuated evoked negative seen in the control. At least in GL, this positive wave may be activity mediated antidromically in geniculocortical fibers. This response tended to improve in form and amplitude with shorter C-T times and no occlusive interaction could be readily demonstrated.

Comparing these interactions with those in the upper right frame where a 5 -msec photic flash was the conditioning stimulus and the cortical shock was the test stimulus, one sees in the depth loci a different picture. No positive wave appears and there is minimal blocking effect. At the surface, however, the IDRs are 100 per cent blocked and the effect in suprasylvius strongly suggests true inhibition since the photic flash evokes minimal activity.

It was mentioned above that TCRs appeared at both cortical locations, but in the interaction frames the early wave in marginalis can now be seen to be untypical of a TCR, since a considerable amount of block of this very early event is now apparent. As will be noted in other figures below, this particular early wave is unusual. In this preparation the true form of the wave is distorted by the stimulus artifact overshoot.?

A late wave to flash at $105 \mathrm{msec}$ to the peak positive in this experiment will be discussed.

In another experiment (Fig. 2), occlusive interaction is seen at much longer $\mathrm{C}-\mathrm{T}$ intervals. Responses at the cortex to mesencephalic reticular

2 Suprathreshold stimulation frequently produced large stimulus artifact overshoots which could not always be simultaneously balanced out on the four different channels. Preamplifier bandpass was 0.8 to $2,000 \mathrm{cycle} / \mathrm{sec}$. 
stimulation ("D") and the IDR to cortical stimulation ("C") are interacted at $300 \mathrm{msec}$ ("DC") with complete IDR block. This was the limit of 100 per cent blocking effect. On the right of this figure is seen the reciprocal effect of the IDR on the response at the same cortical recording electrode, following depth stimulation. This block was complete at C-T of $155 \mathrm{msec}$ and about 50 per cent at $220 \mathrm{msec}$. In this particular experiment, the blocking effect of the conditioning shock to the depth locus was especially prolonged and powerful.

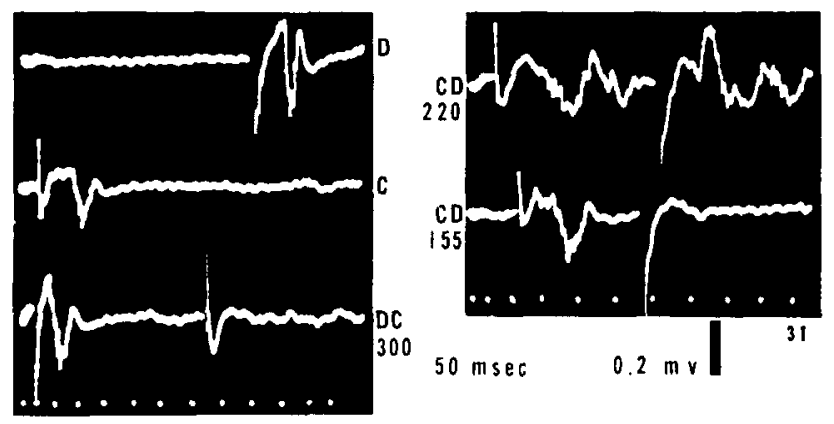

FIG. 2. Cortex and mesencephalic interactions. Cortical stimulation of left gyrus mid-suprasylvius, rccordings from left mesencephalic reticular formation (A2.5, L3.5, -1.75 , histologically verified) and right posterior gyrus marginalis, lateral edge. $D$ is cortical response to reticular stimulation, $\mathrm{C}$ is cortical response (at same electrode as D) to contralateral cortical stimulation. $\mathrm{DC}$ and $\mathrm{CD}$ are interactions at C-T millisecond-intervals as indicated. Posterior commissure and two-thirds of corpus callosum posteriorly, cut several weeks previous to acute experiment.

Responses elicited from the majority of depth loci studied usually did not have as much blocking effect on the IDR as did conditioning shocks to the cortex (dual IDR shocks). In a sense, then, the depth loci may be thought to have varying degrees of access to the cortical fields normally discharging the IDR.

Curves depicting interaction between a mesencephalic reticular location and the ipsilateral and contralateral cortically-elicited IDR (Fig. 3; another experiment), show the effective C-T interval for dual cortical shocks to be twice that of the situation wherein MR stimulation is either test or conditioning. These data are in agreement with the consistent finding that it is virtually imposssible to interact cortical potentials evoked by stimulation at any given brain-stem locus with the surfaceelicited IDR as effectively as can be done by employing afferent stim. 
ulation (e.g., photic and cortical shock interactions) or strictly cortical stimulation (dual cortical shocks). Depth relays and those structures with access to the cortical fields discharging the IDR must have diffuse afferent and efferent projections making definition of distinctive pathways difficult.

The following structures have been adequately studied to enable us to conclude, by our criteria, that they have access in some manner to the IDR more direct pathway in the brain stem, or can, by some other as yet undefined route, interact with the IDR cortical events. These are

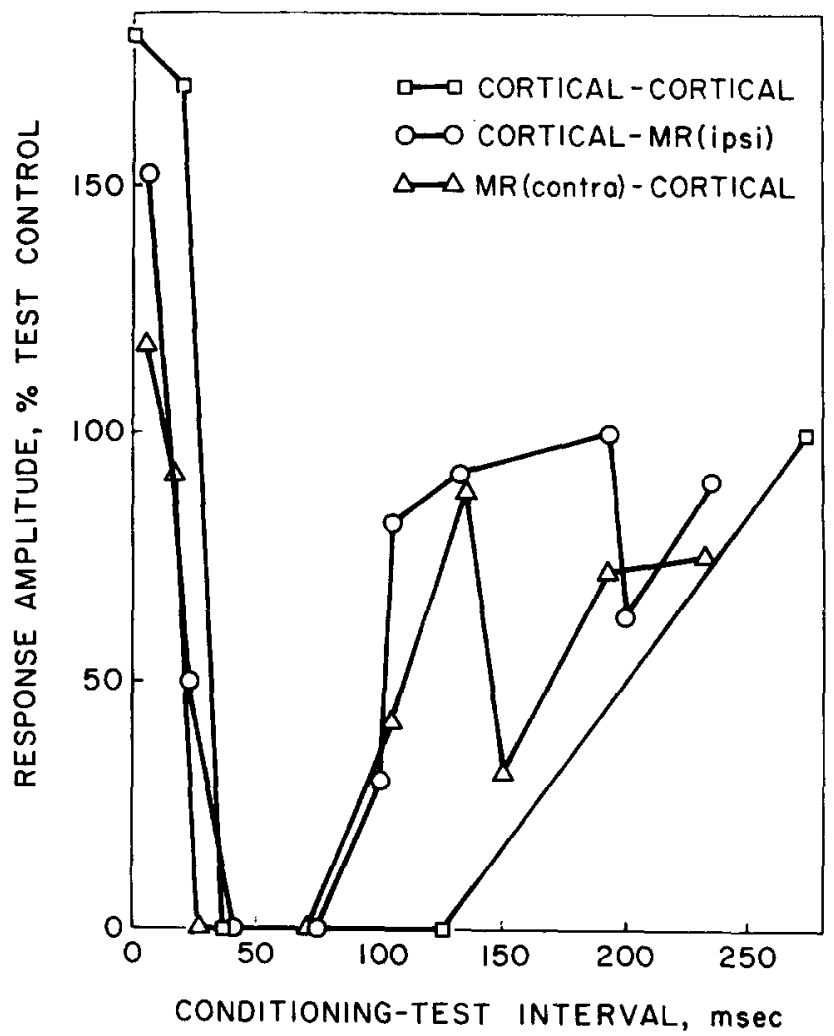

FIg. 3. Extent of interactions. Homologous cortical stimulating and recording loci in mid-suprasylvius. Cortical-cortical: conditioning and test shocks to left cortex, record right. Cortical-MR(ipsi) : conditioning and test shocks to left cortex and left mesencephalic reticular formation histologically verified at $\mathrm{A} 3, \mathrm{~L} 3.5,-2$, record right. MR(contra)-cortical: conditioning shock to left mesencephalic reticular formation, test shock to right cortex, record left. 
the superior colliculus, pulvinar, internal capsule, n. centrum medianum, $\mathrm{n}$. lateralis posterior, medial geniculate, lateral geniculate, interpeduncular nucleus, and $\mathrm{n}$. centralis medialis. In any given experiment interaction curves involving these structures are so similar to those involving $M R$ and cortex (e.g., see Fig. 3) that no additional plottings are necessary.

Summation. Besides interaction of depth and cortically elicited responses, summation of subthreshold stimuli yielding responses similar to those evoked by suprathreshold stimuli have been observed.

The lower left and middle frames in Fig. 4 show cortical responses and
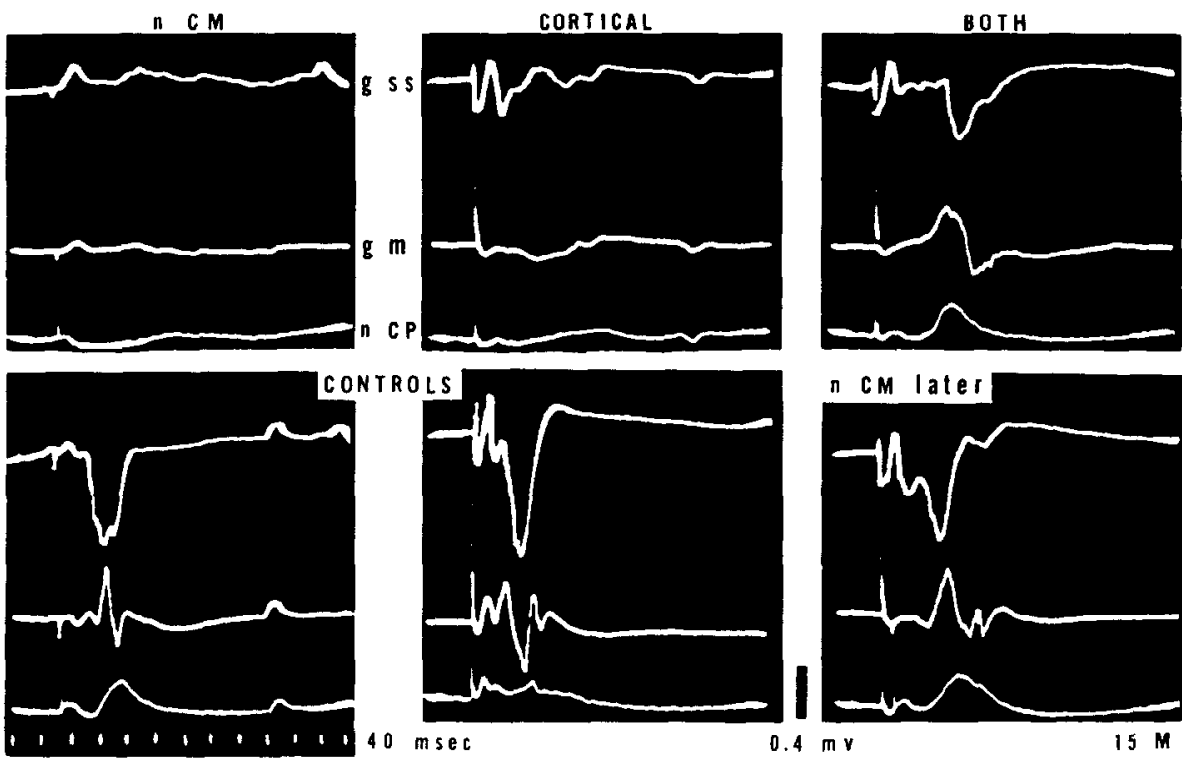

Fig. 4. Centralis medialis-cortical summation. Left column: subthreshold (4 volts, $0.1 \mathrm{msec}$ ) and suprathreshold ( 9 volts, $0.1 \mathrm{msec}$ ) stimulation of $\mathrm{n}$. centralis medialis (n CM) histologically verified at $\mathrm{A} 9, \mathrm{~L} 1.5,-0.5$. All recordings on right gyri suprasylvius and marginalis ( $\mathrm{g}$ ss and $\mathrm{g} \mathrm{m}$ ) and nucleus of posterior commissure ( $\mathrm{n} \mathrm{CP}$ ). Middle column: subthreshold ( 14 volts, $0.5 \mathrm{msec}$ ) and suprathreshold (30 volts, $0.5 \mathrm{msec}$ ) of left suprasylvius. Right column, upper: $\mathrm{n}$ CM and cortical subthreshold stimulation simultaneously; lower: same, but n CM shock 20 msec after cortical.

depth responses in nucleus of posterior commissure (n CP) to suprathreshold nucleus centralis medialis ( $\mathrm{CM}$ ) and cortical stimulation. Components of the TCR are readily distinguishable in gyri suprasylvius and marginalis, whereas the IDR in the latter is more complex with a 
smaller positive wave. The response in $\mathrm{n} C P$ following cortical stimulation is very small and attenuated and may be simply a representation of events occurring in nearby MR. Extent of similarity of the responses to $\mathrm{n} C M$ stimulation can be compared in the lower left frame. In the upper left and middle frames are "responses" to subthreshold stimulation of $\mathrm{n} \mathrm{CM}$ and cortex, except that a part of the TCR is seen in gyrus suprasylvius. When these subthreshold stimulations are combined (upper right) there is a summation which produces evoked activity resembling that in response to suprathreshold stimulation. If $\mathrm{n} \mathrm{CM}$ stimulation is delayed $20 \mathrm{msec}$ (lower right frame) there is a distinct improvement in wave form of the summated response. In this experiment the evoked response in $\mathrm{n} \mathrm{CM}$ to cortical stimulation was nearly nonexistent and certainly smaller than that in $\mathrm{n}$ CP. Therefore, it must be concluded that this summation occurs at the cortex and that $\mathrm{n} \mathrm{CM}$ is not involved in the interhemispheric relay of the IDR.

Negative Brain Stem or Other Areas. Certain areas when stimulated could neither influence the cortically-elicited IDR (infrequently an erratic response completely independent of the IDR could be evoked at the cortex) nor could cortical stimulation produce a response in the structure similar to a relay area response. Occasionally a structure (e.g., hippocampus) would yield a response but unrelated to the IDR. Most of these responses, for example that of hippocampus, had a latency of $10 \mathrm{msec}$ or less, in contrast to the slow waves from "indirect" structures or the latency of the IDR itself. As studied, n. ventralis anterior, anterior thalamic radiations, $\mathrm{n}$. ruber, $\mathrm{n}$. medialis dorsalis, mammillary bodies, mamillo-thalamic tract and posterior hypothalamus, n. rhomboidens, $\mathrm{n}$. reuniens, Z1 Field of Forel, fornix and corpus callosum, and hippocampus all fall into this "negative" category. The preoptic area and the caudate are also probably negative.

Split-Brain Preparations. The data so far discussed were obtained from cats with intact neural structures, the effort being to determine how the IDR is relayed to the contralateral hemisphere. The nonessential nature of structures anterior to the MR in this relay became apparent only with testing of five animals previously prepared according to the technique of Magni, Melzack, and Smith (8). In this procedure sterentaxically oriented needles located at the anterior and posterior extents of the proposed cut carry a piece of suture material in their eyes to the desired depth, the thread is then pulled taut between the eyes severing the intervening tissue. Our animals were originally prepared for be- 
havioral studies, several months previous to their use here. In all five, when studied acutely, the IDR has been seen and many of the features discussed in previous sections have been demonstrated.

The intention was to split in the mid-line to the base of the brain from the anterior pole back into the mesencephalon, from the genu of the corpus callosum posterior to include the splenium and to horizontal +0 . Figure 5 is a composite drawing of the histology of one of these brains. The major findings were complete separation or destruction of the corpus callosum, anterior and posterior commissures, fornix, septum, massa intermedia, and superior colliculus. Additional damage extended 2 or $3 \mathrm{~mm}$ beyond the desired cut in several places.

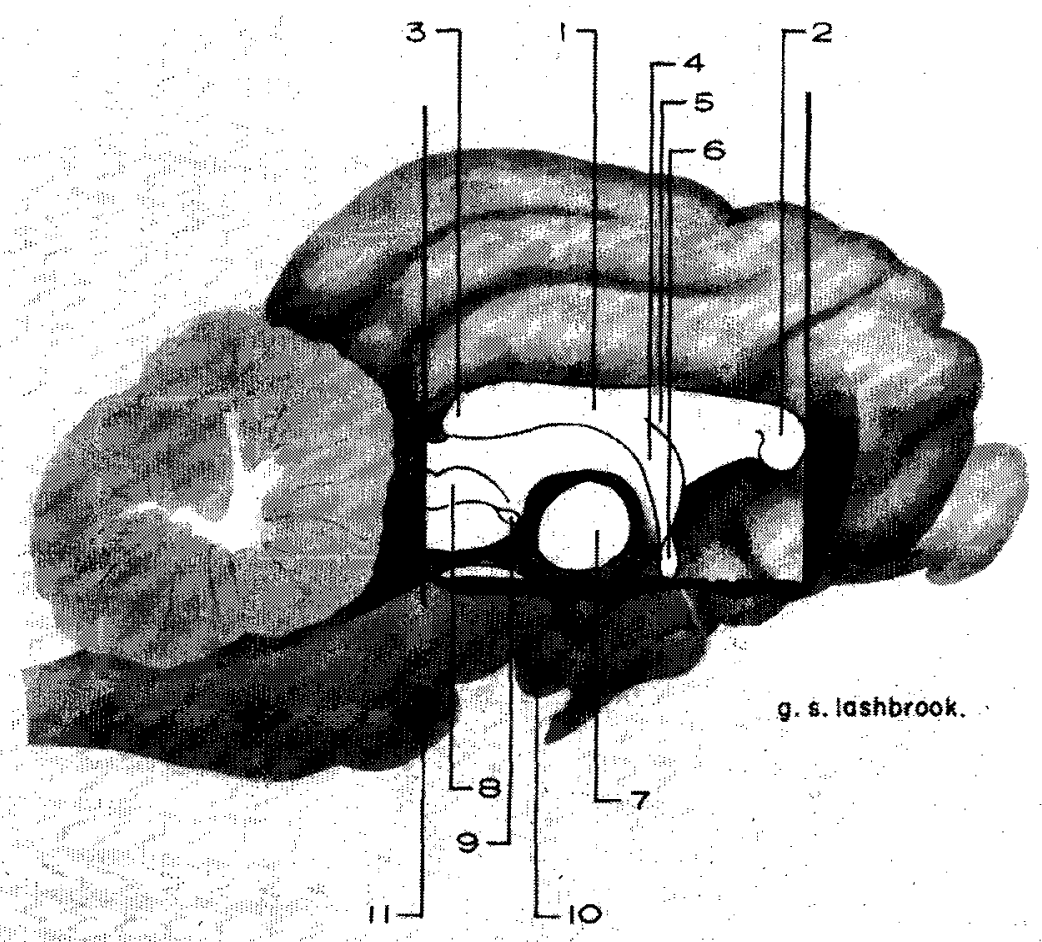

FIG. 5. Extent of cut in split brain. Drawing composed from medial longitudinal celloidin sections, cell body, and fiber stains. Shaded areas outside central cut represent additionally damaged tissue, roughly equal in both hemispheres. Area numbered 1 , corpus callosum (body) ; 2, genu of corpus callosum; 3 , splenium; 4, septum pellucidum; 5, pillars of fornix; 6, anterior commissure; 7 , massa intermedia; 8 , corpus quadrigeminum; 9 , posterior commissure; 10 , mammillary body; 11 , pons. 
Figure 6 shows some of the results obtained in the acute cat of Fig. 5. On the left are superimposed and "representative" single sweep responses to contralateral surface stimulation evoked at two cortical sites, as indicated. Suprasylvius responses, as usual in the acute cat, are of a higher amplitude and more stable than on gyrus marginalis. Variability and

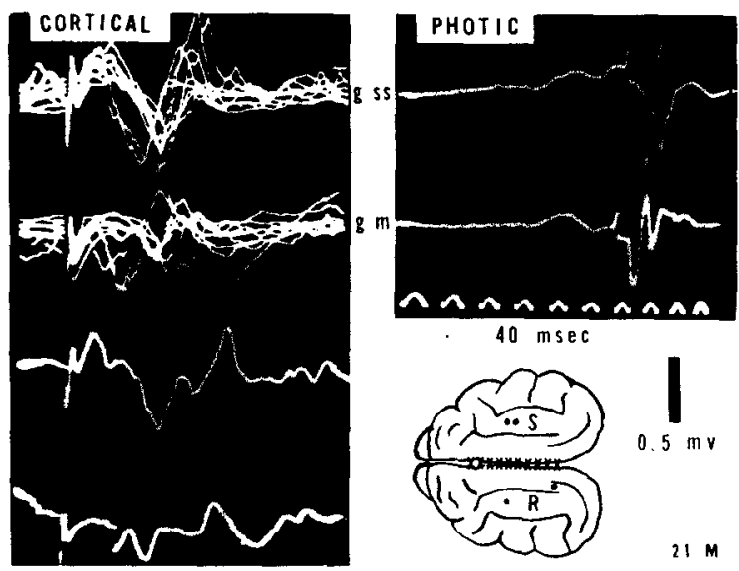

FIG. 6. Split brain: IDR and photic evoked potentials; $50 \mathrm{mg} / \mathrm{kg}$ chloralose and Flaxedilized. Left: right gyrus suprasylvius stimulation ( $S$, inset), recording from contralateral suprasylvius and gyrus marginalis ( $\mathrm{g}$ ss and $\mathrm{g} \mathrm{m}, \mathrm{R}$ on inset) superimposed and selected single frames. Right: responses to 5-msec flash at same recording electrodes.

waxing and waning characterize the IDR in this as in the intact preparation. In suprasylvius at least, the response has distinct triphasic components, though the positive wave is largest. From this figure, one must conclude in part that the remaining mesencephalic structures are able to relay the electrical events between the cortical hemispheres. One should not, however, rule out the suspicion that conduction in the IDR system may have been facilitated in the "neural readjustment" likely to occur during the nearly 5 months following most of the operations. This possibility is now under investigation.

Finally, as a control, single sweeps of evoked potentials to flash from the same recording electrodes as at left, are shown in the right hand frame in Fig. 6. An excellent primary potential, positive-negative, is observed at both locations and is of even higher amplitude on suprasylvius. Again (Fig. 1), a "second sequence," consisting mostly of a positive wave, is 
seen at both electrodes, following closely the negative of the primary potential. As established in other experiments not reported here, this is not an "off" response to the 5 -msec flash. Latency to peak of the positive wave is 60 to $90 \mathrm{msec}$ with the shorter values appearing in association cortex and longer in primary visual cortex. In this experiment cortical and photic stimulation were interacted in much the same fashion as in Fig. 1. Though not shown in these figures, it is the so-called second sequence of the evoked potential complex to photic flash which interacts with the IDR and thus suggests common cortical fields.

\section{Discussion}

With the plethora of excellent reports appearing in recent years on the critical role of the brain-stem reticular core in neural functions, it should not be surprising that interhemispheric pathways linking cortical regions may pass through this area. Up to now, experimental data have not established a firm basis for such pathways. More than 10 years ago corticofugal electrophysiological responses were found in brain-stem areas, especially in the mesencephalic reticular formation and the surrounding area (6). However, neither electrophysiological evidence, nor anatomical $(5,10)$, have shown really strong projections from association cortex (gyrus suprasylvius in the cat) which when stimulated generally elicits IDRs of largest amplitude. Those areas with well-defined corticofugal projections to the brain stem (e.g., sensorimotor) are those not likely involved in the IDR since their stimulation is relatively ineffective in discharging IDR waves on the contralateral hemisphere. It is certain that the IDR route is indirect, with many synapses. It is also likely that the corticofugal activity projects to intermediate nuclei which function to maintain a relative synchrony of discharge into the mesencephalic ret:cular formation.

Projections between cortex and specific and nonspecific thalamic nuclei have been shown by a number of workers to mediate such responses as the augmenting, repetitive, and recruiting potentials. Under certain conditions these waves were seen to be mediated by interhemispheric connections. For example, as evidence of the importance of mid-line thalamic nuclei in the interhemispheric conduction of the recruiting response, Enomoto (3) showed the critical nature of the anterior portion of the massa intermedia. The study of the IDR in the split-brain preparation certainly rules out an essential role for mid-line thalamic structures in the IDR relay. In addition, the detrimental effects of barbiturates and 
fast repetitive stimulation, the lability, and the cortical distribution, lead one to conclude that the IDR, though superficially resembling other "nonspecific-type"" or even so-called "artifactual" (9) responses, probably represents a different system.

Mention should be made again of the role of chloralose in the study of IDR characteristics. We wish to entertain seriously the possibility that chloralose in light to moderate doses acts in some systems to block neurons the role of which is normally that of inhibition. Recent data from spinal cord investigations lend weight and significance to this explanation. Dr. J. Haase in Göttingen has discovered that chloralose in low concentrations acts specifically to block Renshaw-cell discharge. ${ }^{3}$ However, inhibitory cells of this type have yet to be found at supraspinal levels. Another suggestion which lacks any supportive evidence at present is that chloralose, again in light to moderate doses, may act directly on cell membranes as a disinhibitor, by producing degrees of hypopolarization.

The recent reports on the "association" response of Buser and colleagues $(1,2)$, among others successfully employing chloralose in supraspinal investigations, point to the likelihood that our IDR system is intimately related to conduction and elaboration in their association areas. As with the IDR, the "association" response can be seen in the unanesthetized preparation but is more easily investigated under chloralose. It is considered highly likely, too, that the late wave seen following the primary evoked potential to flash (Fig. 6) is probably identical with these investigators' "association" response. This late wave to flash is certainly related to the IDR, for in preliminary experiments it could be reciprocally interacted with the IDR and evidenced similar refractoriness.

Our interaction studies have shown that a somewhat diffuse relay in the mesencephalon and certain other brain-stem areas, which are not activated by cortical stimulation as a part of the IDR system and are less effective in evoking cortical waves, have access to portions of the cortical fields discharging the IDR. Reciprocal interaction data point to modes of cortical and brain-stem control based in part on interstimuli time relations. Other related factors, such as degree of "habituation," frequency and intensity of afferent input, stage of waxing or waning, and the state of spontaneous cortical activity, must help determine the "significance" of an integrated neural process. Some roles of brain-stem structures in mediating neural activity are recognized. Since the work of Starzl,

3 Personal communication. 
Taylor, and Magoun (14), it has been adequately demonstrated electrically that peripherally initiated afferent events of a wide variety can activate neurons in the brain-stem reticular core. Electrical stimulation in this core can in turn evoke widespread cortical events, and thus implications of the importance for behavior of reticular-cortical-reticular interactions become apparent.

The above findings and interpretations lead to a consideration of the possible functional significance of an electrically conducting neural system linking the two cortices by way of a mesencephalic reticular relay. Sperry (13), in a review of his and others' work, has discussed the type of information which is unlikely to be relayed between the hemispheres in the split-brain preparation. Indeed, a split as radical as in our cats yields a chronic preparation which under given experimental conditions and with important exceptions, functions as if it had two brains! It is yet to be demonstrated whether these exceptions and other experimental circumstances will suggest a behavioral role for the IDR system. If they do, possibly interhemispheric transfer of some learned behaviors can be blocked or facilitated by interfering with IDR conduction.

\section{References}

1. Buser, P., and P. Borenstein, Résponses somesthésiques, visuelles et auditives, recueillies au niveau du cortex "associatif" suprasylvien chez le chat curarisé non anesthésié. Electroencephalog. and Clin. Neurophysiol. 11: 285-304, 1959.

2. Buser, P., P. Borenstein, and J. Bruner, Etude des systèmes "associatifs" visuels et auditifs chez le chat anesthésié au chloralose. Electroencephalog. and Clin. Neurophysiol. 11: 305-324, 1959.

3. Eмомото, T. F., Unilateral activation of the non-specific thalamic system and bilateral cortical responses. Electroencephalog. and Clin. Neurophysiol. 11: 219-232, 1959.

4. Feldberg, W, J. L. Malcolm, and I. D. Smith, Effect of tuborcurarine on the electrical activity of the cat's brain under chloralose. J. Physiol. London 138: 178-201, 1957.

5. GobBel, W. G., JR., and G. W. Liles, Efferent fibers in the parietal lobe of the cat. J. Neurophysiol. 8: 257-266, 1945.

6. Jasper, H., C. AJMone-Marsan, and J. Stoll, Corticofugal projections to the brain stem. A.M.A. Arch. Neurol. Psychiat. 67: 155-171, 1952.

7. KING, E. E., Differential action of anesthetics and interneuron depressants upon EEG arousal and recruitment responses. J. Pharmacol. Exptl. Ther. 116: 404-417, 1956.

8. Magni, F., R. Melzack, and C. J. Smith, A stereotaxic method for sectioning the corpus callosum in cat. Electroencephalog. and Clin. Neurophysiol. 12: $517-518,1960$. 
9. Ostow, M., The electrical responses of the cerebral cortex to corticopetal impulses: a comparison of peripheral and contralateral cortical stimulation. J. Mt. Sinai Hosp. N. Y. 21: 226-246, 1954.

10. Rossi, G. F., and A. Brodal, Corticofugal fibers to the brain stem reticular formation. An experimental study in the cat. J. Anat. 90: 42-62, 1956.

11. Rutledge, L. T., and T. T. Kennedy, Extracallosal delayed responses to cortical stimulation in chloralosed cat. J. Neurophysiol. 23: 188-196, 1960.

12. Rutledge, L. T., and T. T. Kennedy, Subcortical relay of the interhemispheric delayed response. Federation Proc. 20: 337, 1961.

13. Sperry, R. W., Cerebral organization and behavior. Science 133: 1749-1757, 1961.

14. Starzl, T. E., C. W. Taylor, and H. W. Magoun, Collateral afferent excitation of reticular formation of brain stem. J. Neurophysiol. 14: 479-496, 1951. 